\title{
Microwave Circuits Based on Negative Refractive Index Material Structures
}

\author{
Christophe Caloz, Atsushi Sanada, and Tatsuo Itoh \\ University of California, Los Angeles, Department of Electrical Engineering, \\ 405 Hilgard Avenue, CA 90095, Los Angeles, USA, 310-206-1710, caloz@ee.ucla.edu
}

\begin{abstract}
The paper presents the fundamentals of composite right/left-handed (CRLH) transmission lines and materials, and proposes an accurate circuit model of these structures for the design of practical applications. Three novel applications of CRLH meta-structures, developed at UCLA, are demonstrated: an arbitrary coupling-level backward-wave coupler, a zero ${ }^{\text {th }}$ order resonator and a distributed planar negative lens.
\end{abstract}

\section{INTRODUCTION}

Recently, there has been significant interest for novel metamaterials, and in particular for left-handed (LH) materials, which are characterized by antiparallel-phase and group velocities [1]-[2]. The novelty of the physics related with these artificial structures may lead to revolutionary applications in optical and microwave devices and materials.

Our group at UCLA has been using a transmission line (TL) approach of metamaterials [3], based on nonresonant low-loss and broad-bandwidth elements, and has introduced the concept of composite right/left-handed structures (CRLH) as a framework for the understanding and design of novel metamaterial applications [4]. This concept is based on the fact that any practical $\mathrm{LH}$ structure, which can be modeled by series $\mathrm{C}$ and shunt $\mathrm{L}$, becomes right-handed ( $\mathrm{RH})$ as frequency increases due to the presence of natural $\mathrm{RH}$ parasitic series $\mathrm{L}$ and shunt C.

This paper will first present the fundamentals of CRLH TLs. Then it will describe how to model them. Finally, it will demonstrate three selected applications developed at UCLA.

\section{CRLH LINES AND MATERIALS}

An ideal CRLH-TL is represented in Fig. 1 by its circuit model. The most appropriate model in practice is the one of Fig. 1a, which has the dispersion relation

$$
\beta=\sqrt{\omega^{2} L_{R}^{\prime} C_{R}^{\prime}+\frac{1}{\omega^{2} L_{L}^{\prime} C_{L}^{\prime}}-\left(\frac{L_{R}^{\prime}}{L_{L}^{\prime}}+\frac{C_{R}^{\prime}}{C_{L}^{\prime}}\right)},
$$

from which all the parameters $Z_{0}, v_{p}, v_{g}, t_{g}$ can be straightforwardly derived in a closed form. However, it can be easily seen that in the particular balanced case, defined as

$$
L_{R}^{\prime} C_{L}^{\prime}=L_{L}^{\prime} C_{R}^{\prime}=L^{\prime} C^{\prime},
$$

the model of Fig. 1a is equivalent to the model of Fig. 1b, which is simpler and provides a more direct insight into the physical properties of CRLH structures [4]. The corresponding dispersion relation,

$$
\beta_{s}=\beta_{R}+\beta_{L}=\omega^{2} L_{R}^{\prime} C_{R}^{\prime}-\frac{1}{\omega^{2} L_{L}^{\prime} C_{L}^{\prime}},
$$

emphasizes the dual nature of metamaterials, which exhibit LH characteristics at low frequency and RH characterisitics at high frequencies, with a transition frequency given in general by

$$
\omega_{0}=\frac{1}{\sqrt[4]{L_{R}^{\prime} C_{R}^{\prime} L_{L}^{\prime} C_{L}^{\prime}}} \stackrel{\text { balanced }}{=} \frac{1}{\sqrt{L^{\prime} C^{\prime}}},
$$

where $\beta=0$, and therefore $\lambda_{g}=\infty$. If the structure is unbalanced $\left(L_{R}{ }^{\prime} C_{L}\right.$ ' $\neq L_{L}{ }^{\prime} C_{R}$ '), and therefore mismatched (Fig. 1b), a gap will open up in some frequency range, due to the negative sign appearing in (1); in this case, (4) can be shown to represent the frequency of highest attenuation in the gap. It can also be shown from the model of Fig. 1b that $v_{g}$ does not tend to $\infty$ (as suggested by a purely $\mathrm{LH}$ model) when $\omega \rightarrow \infty$, but to $1 / \sqrt{ }\left(L_{R}{ }^{\prime} C_{R}{ }^{\prime}\right)$ $=c_{0} / n$ (no more RH effect).

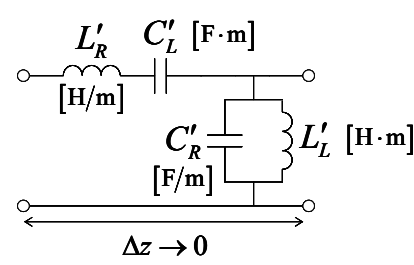

(a)

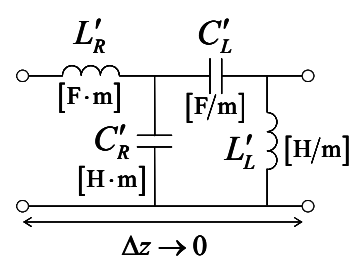

(b)

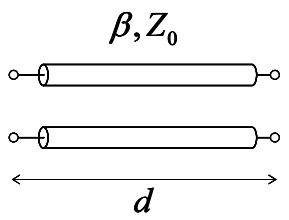

(c)

Fig. 1 Ideal CRLH-TL. (a) Infinitesimal circuit model. (b) Equivalent infinitesimal circuit model in the balanced case $\left(L_{R}{ }^{\prime} C_{L}{ }^{\prime}=L_{L}{ }^{\prime} C_{R}{ }^{\prime}\right)$. (c) Line representation and physical parameters. The subscripts $\mathrm{R}$ and $\mathrm{L}$ stand for $\mathrm{RH}$ and $\mathrm{LH}$, respectively.

The ideal structure of Fig. 1 can be synthesized by repeating periodically (convenient but not necessary) an 
electrically small unit cell with inductances/capacitances $L_{R} / C_{L} / C_{R} / L_{L}(\mathrm{H} / \mathrm{F})$ mimicking the incremental cell of Fig. 1a. The corresponding dispersion relation can be computed analytically by using Kirchoff's laws and Bloch-Floquet theorem (also possible in 2D [5] or 3D). This relation is given by

$$
\cos (\beta a)=1-\frac{1}{2}\left[\frac{1}{\omega^{2} L_{L} C_{L}}+\omega^{2} L_{R} C_{R}-\left(\frac{L_{R}}{L_{L}}+\frac{C_{R}}{C_{L}}\right)\right]
$$

and plotted in Fig. 2 .

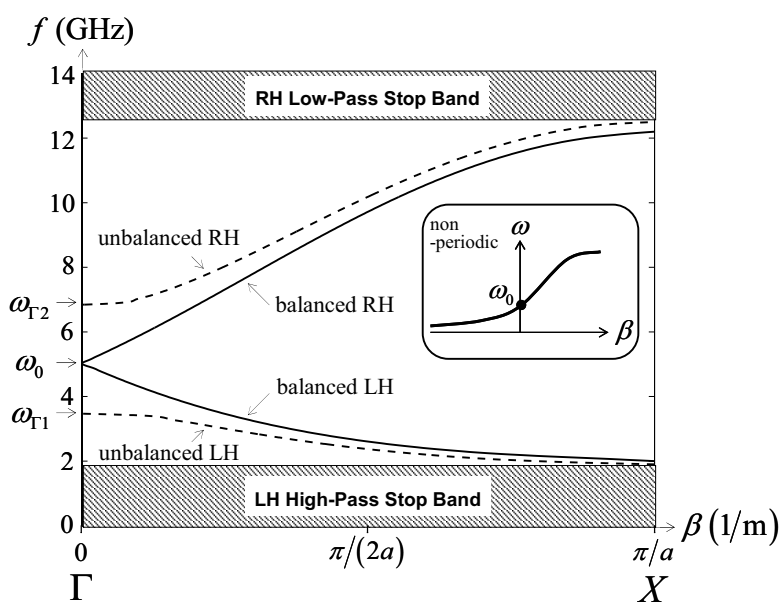

Fig. 2 Dispersion relation computed for the balanced and unbalanced CRLH-TL. Balanced: $L_{R}=L_{L}=1 \mathrm{nH}, C_{R}=C_{L}=1$ pF; unbalanced: $L_{R}=1 \mathrm{nH}, L_{L}=5.5 \mathrm{nH}, C_{R}=1 \mathrm{pF}, C_{L}=2 \mathrm{pF}$. The inset shows the dispersion curve of a balanced non periodic CRLH-TL.

\section{PARAMETERS EXTRACTION}

An accurate parameters extraction of CRLH structures is crucial for efficient design. The first two applications of the next section use a microstrip implementation of the CRLH structure, as shown in Fig. 3. This implementation was also successfully used in a novel backfire-to-endfire leaky-wave antenna presented in [6].

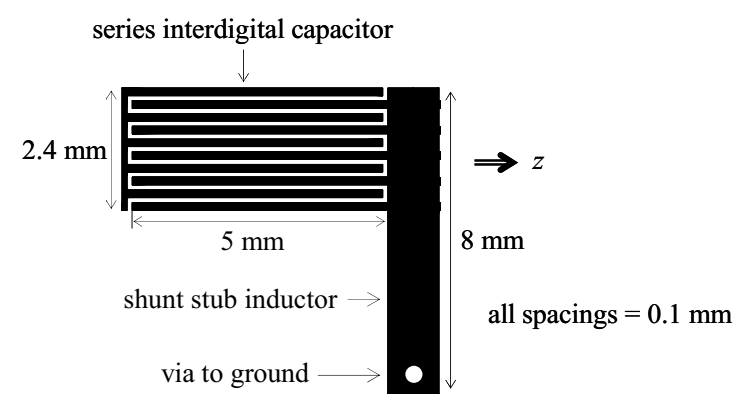

Fig. 3 Layout of the unit cell of the microstrip implementation of the CRLH-TL, including a series interdigital capacitor of value $C_{L}$ and a shunt stub inductor of value $L_{L}$ shorted to the ground plane by a via.

The extraction procedure can be performed with the help of Fig. 4. It consists in the following steps: (1) full- wave simulate or measure, separately, the interdigital capacitor and the stub inductor; (2) transform their Sparameters into $Y$ (for $\mathrm{C}$ ) and $Z$ (for $\mathrm{C}$ ) parameters, whose matrixes are known for the $\Pi$ (for $\mathrm{C}$ ) and $\mathrm{T}$ (for C) networks; (3) then all the values in the top circuits of Fig. 5 are known; (4) finally, obtain the CRLH parameters as

$L_{R}=L_{s}^{\mathrm{int}}, C_{R}=2 C_{p}^{\mathrm{int}}+C_{p}^{\mathrm{stub}}, L_{L}=L_{p}^{\mathrm{stub}}, C_{L}=C_{s}^{\mathrm{int}}$,

where the series inductance of the stub could be neglected.

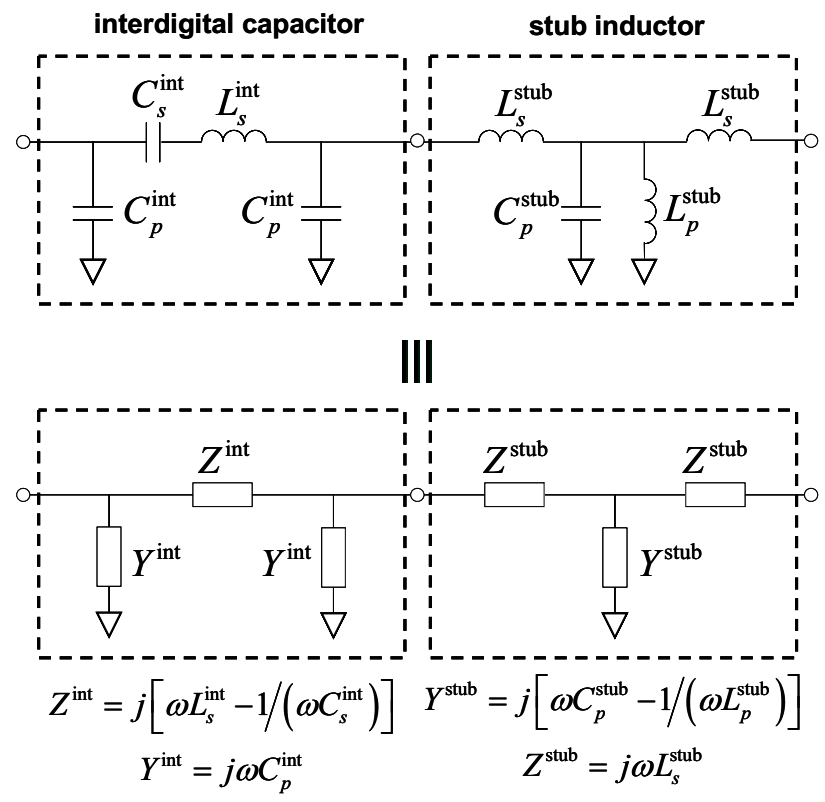

Fig. 4 Circuit model for the unit cell shown in Fig. 3.

\section{SELECTED APPLICATIONS}

\section{A. Arbitrarily Tight Coupled-Line Coupler}

A novel broadband tight backward-wave directional coupler with level [7] is obtained by replacing the microstrip lines of the conventional coupled-line coupler by the CRLH line described in Fig. 3. A rigorous even/odd mode analysis revealed that this device exhibits unique properties:

- It can achieve any arbitrary level of coupling. In comparison, conventional backward coupled-line couplers are typically limited to less than $-10 \mathrm{~dB}$ coupling.

- It is based not only of capacitive-electric coupling (conventional), but also on inductive-magnetic coupling.

- Its electrical length $\beta d$, is not 90 degrees (conventional), as in the conventional case, but zero degrees.

- The previous point is a consequence of the fact that the even/odd equivalent TLs are operating in a frequency gap: their characteristic impedances $Z_{0 e} / Z_{0 o}$ are purely imaginary, and $\gamma_{e, o}=\alpha_{e, o}$, where the 
subscripts e and o stand for even and odd, respectively. The coupling coefficient takes then the form

$$
C_{\mathrm{BWD}}=\frac{\left(Z_{0 e}-Z_{0 o}\right) \tanh [\alpha d]}{2 Z_{0}+\left(Z_{0 e}+Z_{0 o}\right) \tanh [\alpha d]} .
$$

- As seen in (6), the coupling level depends now on the attenuation length $\alpha d$ of the even/odd modes $\left(\alpha_{e} \simeq \alpha_{o}\right)$. If $\alpha d$ is large enough (easily achieved because of gap), then $\tanh (\alpha d)=1$, and it can be easily verified that, with the general formula $Z_{0 e} Z_{0 o}=Z_{0}^{2}$, $C_{\mathrm{BWD}}=1$ (complete coupling).

The coupler operates in the LH range of the CRLH lines. Its physical length depends only on the LC loadings, and can be reduced if identical LC values can be obtained on shorter lengths.

Fig. 5 shows the experimental results of a $0-\mathrm{dB}$ prototype. Any arbitrary level of coupling can be obtained by simply decreasing the length or/and increasing the spacing of the coupler. A 3-dB implementation was demonstrated in [7].

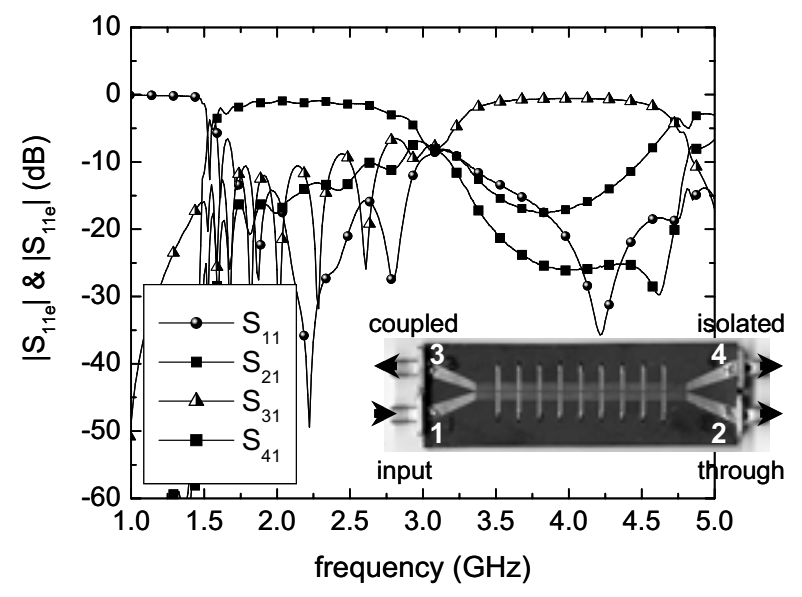

Fig. 5 Measured S-parameters for the a quasi 0-dB $(\sim 0.5 \mathrm{~dB})$ CRLH coupler. The coupler is shown in the inset. The $0-\mathrm{dB}$ coupling bandwidth extends from $3.2 \mathrm{GHz}$ to $4.6 \mathrm{GHz}(\mathrm{BW}=$ $36 \%)$. (spacing $=0.3 \mathrm{~mm})$

\section{A. Zero $^{\text {th }}$ Order Resonator}

As illustrated in Fig. 2, a CRLH structure has a dispersion relation crossing the $\beta=0$ axis at a non-zero frequency $\omega_{0}$ given by (4). This property is exploited in the novel zeroth order resonator [8] presented here.

Fig. 6 illustrates the principle of this resonator. At $\omega_{0}$ there is no phase shift between the input and the output of the structure, since $\theta=\beta d=0$. Therefore, the resonance is flat (uppermost case). Using a mechanical analogy, this mode looks like that of a vibrating string without fixtures (no boundary conditions). Consequently, the resonance is independent of the physical length of the structure, but depends only on its reactive loadings, which determine $\omega_{0}$.
The same microstrip TL as in the coupler was used to design the resonator, which is obtained by simply opening up coupling capacitive slits at both ends of the line. It can be shown that if the line is unbalanced, in the case of capacitive coupling (open-ended) only the shunt antiresonant $C_{R} / L_{L}$ tank resonates, while in the case of inductive coupling (short-ended) it is the series resonant $C_{R} / L_{L}$ tank which resonates.

The unloaded $Q_{s}$ of the open/shorted-ended resonator can be shown to be given by

$$
Q_{0}^{\text {open }}=(1 / G) \sqrt{C_{\mathrm{R}} / L_{\mathrm{L}}}, \quad Q_{0}^{\text {short }}=R \sqrt{C_{\mathrm{L}} / L_{\mathrm{R}}},
$$

where $G$ and $R$ represent the shunt conductance and series resistance of a lossy CRLH-TL, respectively. These $\mathrm{Q}$ factors are seen to be independent of the number of unit cells.

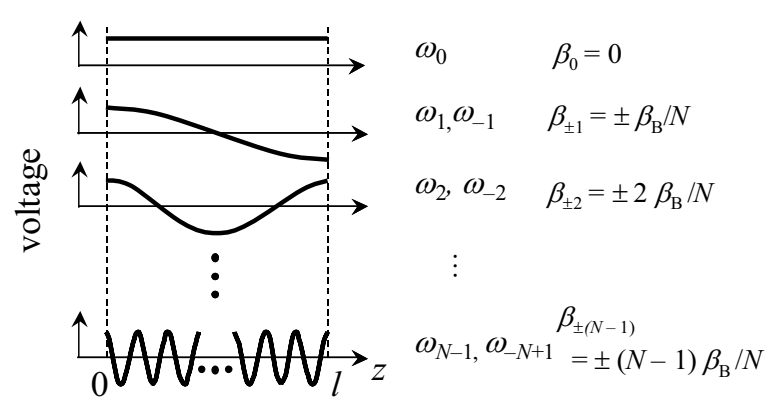

Fig. 6 Open-ended CRLH TL resonator modes. N represents the number of unit cells in the CRLH structure.

Fig. 7 shows the resonance characteristics of a 1-cell CRLH resonator compared with simulation. The measured $Q$ factor of this zero mode is 250 , and the size of the resonator is approximately $\lambda_{0} / 5$.

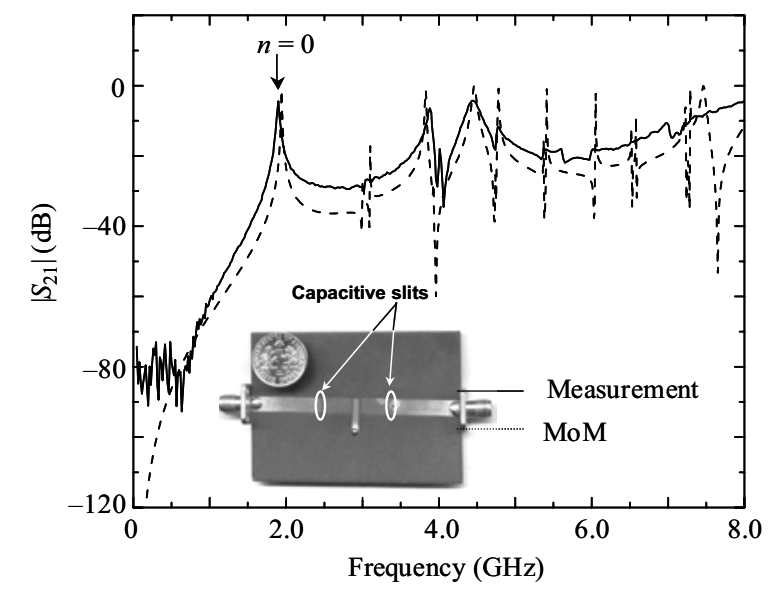

Fig. 7 Measured frequency characteristics of the CRLH TL resonator (solid line). Dashed line is the MoM simulation result. The prototype is shown in the inset.

\section{B. Planar Distributed Negative "Lens"}

The third application deals with a 2D CRLH structure. In [9], Pendry analyzed a LH straight slab lens, first 
proposed by Veselago in [1], capable of focusing energy following Snell's law with negative refractive index in the slab. This phenomenon was experimented in [10] using a 2D array of lumped components, but this implementation is unpractical because it is limited in frequency by the self-resonance of the SMT chips and because it is incompatible with MMIC processes. We have demonstrated first the first time in [5] negative refractive index and subsequent negative focusing in a planar distributed structure, which can be scaled to any frequency and engineered by standard processes. The structure is essentially the mushroom structure described by Sievenpiper et al. in [11], but with series capacitances strongly enhanced by parallel-plate capacitors provided by metal caps just below the mushrooms heads, as shown in Fig. 8a. By strongly enhancing the series capacitance, in agreement with the CRLH principles described in Sec. II, we were able to obtain a broad $\mathrm{LH}$ range in the fundamental mode, while the corresponding curve shown in [11] for the cap-less structure is essentially flat in the corresponding frequency range.

Fig. $8 \mathrm{~b}$ demonstrated the negative lens effects, characterized by a first focus in the LH medium and a second focus in the output RH medium. This effect may be used in a diversity of applications, including beamformers and power combiners.

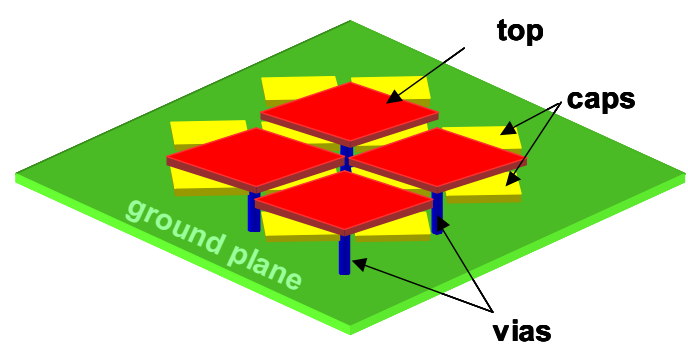

(a)

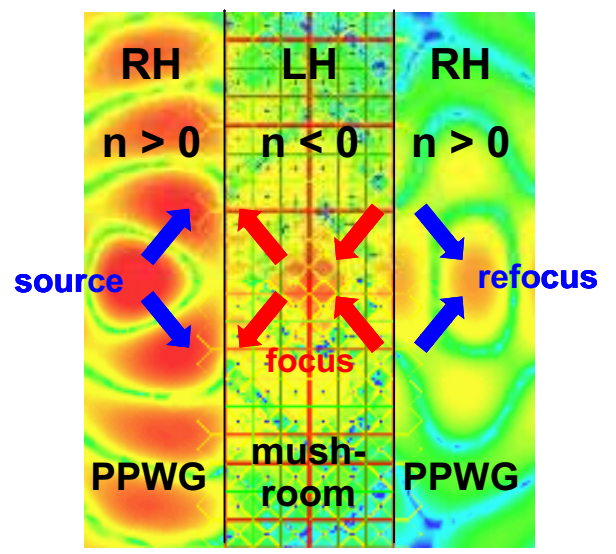

(b)

Fig. 8 (a) Capacitively-enhanced mushroom structure. (b) Full-wave simulated negative lens effect. A mushroom 6 × 19 cells mushroom structure operating in a $\mathrm{LH}$ region of its dispersion diagram is sandwitched between two parallel-plate waveguide (RH) structures. The plot shows the full-wave simulated electric field in the structure. The structure is excited by a vertical coaxial excitation.

\section{CONCLUSION}

The fundamentals of CRLH TLs have been presented and an accurate model of practical CRLH structures has been proposed. Three unique applications of CRLH metastructures developed at UCLA have been demonstrated.

\section{ACKNOWLEDGEMENT}

This work is part of the MURI program "Scalable and Reconfigurable Electromagnetic Metamaterials and Devices". It was supported by the Department of Defense (N00014-01-1-0803) and monitored by the U.S. Office of Naval Research.

\section{REFERENCES}

[1] V. G. Veselago, "The electrodynamics of substances with simultaneously negative values of $\varepsilon$ and $\mu$," Soviet Physics Uspekhi, vol. 10, No. 4, pp.509-514, January-February, 1968.

[2] D. R. Smith, W. J. Padilla, D. C. Vier, S. C. Nemat-Nasser, and S. Schultz, "Composite medium with simultaneously negative permeability and permittivity," Phys. Rev. Lett., vol. 84, no. 18, pp. 4184-4187, May 2000.

[3] C. Caloz, and T. Itoh, "Application of the transmission line theory of left-handed (LH) materials to the realization of a microstrip LH transmission line", IEEE-APS Int'l Symp. Digest, vol. 2, pp. 412415, June 2002.

[4] C. Caloz, and T. Itoh, "Novel microwave devices and structures based on the transmission line approach of meta-materials," IEEEMTT Int'l Symp. Digest, pp.195-198, Philadelphia, PA, June 2003.

[5] A. Sanada, C. Caloz, and T. Itoh, "2D distributed meta-structures with negative refractive properties," IEEE AP-S USNC/URSI Digest, vol. 1, Columbus, OH, June 2003.

[6] L. Liu, C. Caloz and T. Itoh, "Dominant Mode (DM) Leaky-Wave Antenna with Backfire-to-Endfire Scanning Capability", Electron. Lett., vol. 38, no. 23, pp. 1414-1416, November 2002.

[7] C. Caloz, A. Sanada, L. Liu, and T. Itoh, "A Broadband LeftHanded (LH) Coupled-Line Backward Coupler with Arbitrary Coupling Levels", IEEE-MTT Int'l Symp., vol. 1, pp. 317-320, Philadelphia, PA, June 2003.

[8] A. Sanada, C. Caloz, and T. Itoh, "Zero" Order Resonance in Composite Right/Left-Handed Transmission Line Resonators", accepted at Asia-Pacific Microwave Conference, Seoul, Korea, November 2003.

[9] J. B. Pendry, "Negative refraction makes a perfect lens," Phys. Rev. Lett., vol. 85, no. 28, Oct. 2000

[10] G. V. Eleftheriades, A. K. Iyer, and P. C. Kremer, IEEE Trans. on Microwave Theory Tech., vol. 50, no. 12, pp. 2702-2712, Dec. 2002.

[11] D. Sievenpiper, L. Zhang, R. F. J. Broas, N. G. Alexopólous, and E. Yablonovich, IEEE Trans. on Microwave Theory and Tech., vol. 47, no. 11, pp. 2059-2074, Nov. 1999. 